\title{
Static structure factor of liquid parahydrogen
}

\author{
J. Dawidowski, ${ }^{1, *}$ F. J. Bermejo, ${ }^{2, \dagger}$ M. L. Ristig, ${ }^{3}$ B. Fåk, ${ }^{4}$ C. Cabrillo, ${ }^{5}$ R. Fernández-Perea, ${ }^{5}$ K. Kinugawa, ${ }^{6}$ and J. Campo ${ }^{7}$ \\ ${ }^{1}$ Consejo Nacional de Investigaciones Científicas y Técnicas, Centro Atómico Bariloche and Instituto Balseiro, Comisión Nacional \\ de Energía Atómica, Universidad Nacional de Cuyo, (8400) Bariloche, Argentina \\ ${ }^{2}$ Department of Electricity and Electronics, University of the Basque Country, P.O. Box 644, E-48080 Bilbao, Spain \\ ${ }^{3}$ Universität zu Köln, Institut für Theoretische Physik, Zülpicher Strasse 77, 50937 Köln, Germany \\ ${ }^{4}$ Département de Recherche Fondamentale sur la Matière Condensée, SPSMS, CEA, F-38054 Grenoble, France \\ ${ }^{5}$ Consejo Superior de Investigaciones Científicas, Instituto de Estructura de la Materia, Serrano 123 E-28006 Madrid, Spain \\ ${ }^{6}$ Department of Chemistry, Nara Women's University, Nara 630-8506, Japan \\ ${ }^{7}$ Instituto de Ciencia de Materiales de Aragón, Consejo Superior de Investigaciones Científicas, Zaragoza, Spain
}

(Received 29 July 2003; published 22 January 2004)

\begin{abstract}
The single-differential neutron-scattering cross section of liquid parahydrogen has been measured at $15.2 \mathrm{~K}$ and 2 bars of applied pressure by means of low-energy neutron diffraction. Our experimental conditions enable the direct observation of the peak of the liquid structure factor and therefore largely improve the signal-to-noise ratio with respect to measurements carried out using higher-energy neutron diffraction. This avoids the need of performing corrections of approximate nature to the measured cross section that is dominated by molecular rotational components if measured by conventional neutron diffraction.
\end{abstract}

DOI: 10.1103/PhysRevB.69.014207

PACS number(s): 61.20.-p, 61.12.-q, 78.70.-g

\section{INTRODUCTION}

Our interest in the basic microscopic properties of condensed molecular hydrogen (solid, liquid, or highly compressed fluid) stems from widely different origins that stretch from astrophysics (it is known to be one of the dominant constituents of the giant planets $^{1}$ ) to basic condensed-matter physics where efforts to cross the insulator-to-metal transition resulting in the production of metallic hydrogen continue apace. ${ }^{2}$

In contrast, the basic quantity $g(r)$ (the radial pair distribution) that is related to many thermodynamic functions for a liquid has not yet been accurately determined for liquid para- $\mathrm{H}_{2}$. While neutron diffraction is the prime experimental technique for the purpose just referred to, there exist a number of serious difficulties that hinder a measurement of the static structure factor $S(Q)$ on an absolute scale. Such difficulties arise from the light masses of its constituent particles and the relatively low temperatures where the liquid exists under its saturated vapor pressure. This makes quantum effects prominent, and its first manifestation is the appearance of a discrete spectrum of transitions between molecular rotational levels. The quantum nature of such motions imposes some symmetry constraints on the total molecular wave function. This means that the rotational states and the nuclear-spin states of the two protons forming the $\mathrm{H}_{2}$ molecule will not be independent. Coupling of nuclear-spin states $(I=0$ for a molecule having antiparallel proton spins and $I=1$ for parallel spin states) leads to two distinguishable species, para- $\mathrm{H}_{2}$ and ortho- $\mathrm{H}_{2}$, respectively.

In addition, it is known from the early days of neutron scattering ${ }^{3}$ that the cross section for liquid hydrogen is extremely sensitive to the incident neutron energy $E_{0}$ and, in fact, for $E_{0}>80 \mathrm{meV}$ there is basically no distinction between the total scattering cross sections of normal and pure para- $\mathrm{H}_{2}$ since the scattering becomes dominated by molecular rotational para $\rightarrow$ ortho transitions. The latter are known to follow molecular form factors that exhibit a wave-vector dependence ${ }^{4}$ rather different from that corresponding to the liquid-structure factor $S(Q)$. The strength of such rotational contribution nearly doubles that comprising the liquid static structure and consequently it dominates the $d \sigma / d \Omega$ accessible to conventional diffraction using hot or epithermal neutrons.

A previous attempt to derive an estimate for $S(Q)$ (Ref. 5) from an inelastic-scattering experiment yielded a value for its height well in excess of that predicted from path-integralcentroid-molecular-dynamics (PICMD) simulations. ${ }^{6}$ Here we report on an experiment conducted using a cold neutron diffractometer that allows us to explore $d \sigma / d \Omega$ under conditions where most of the scattering arises from liquid structure effects rather than single-particle molecular rotations. Contrary to our previous measurement which was not designed for structure determination, the present experiment allows us to measure $d \sigma / d \Omega$ on absolute units from where an estimate of $S(Q)$ for $Q$-values comprising the liquid diffraction peak is derived. On the other hand, under the experimental conditions we are forced to use, a large part of the scattering is inelastic, thus making the usual approximations employed to analyze experimental data to break down. This is a consequence of the closeness of the incident neutron energy to that required to excite a longitudinal phonon. In consequence, extreme care has been taken to apply nonstandard inelasticity corrections that are described in detail in the coming sections.

\section{EXPERIMENT}

The neutron measurements were performed at D1B spectrometer (ILL, Grenoble) at a temperature of $15.2 \mathrm{~K}$ and 2 bars of applied pressure. The sample was obtained from high-purity hydrogen gas transformed to para- $\mathrm{H}_{2}$ by forcing it to pass through an activated catalyst, in a similar procedure as described in a previous paper. ${ }^{7}$ The sample holder was an 


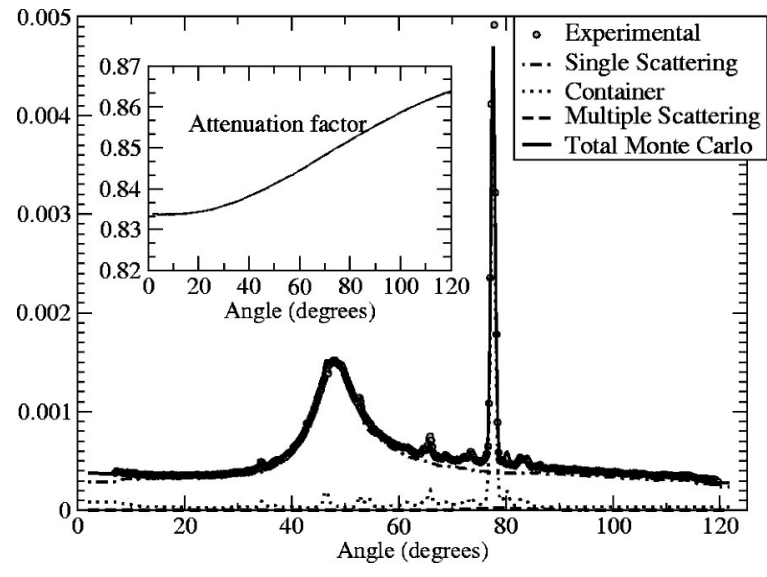

FIG. 1. Experimental raw data (circles) compared with the different components of the Monte Carlo simulation. In the inset, the attenuation factor is shown.

aluminum cylinder, $7 \mathrm{~mm}$ in diameter and $30 \mathrm{~mm}$ high. A vanadium cylinder $10 \mathrm{~mm}$ in diameter and $50 \mathrm{~mm}$ in height was employed to calibrate the absolute scale.

The incident neutrons' wavelength was $2.52 \AA\left(E_{0}\right.$ $=12.88 \mathrm{meV}$ ), which allowed us to cover the range from 0.3 to $4 \AA^{-1}$ in elastic $Q$. In Fig. 1 we show the experimental results after background subtraction.

\section{DATA PROCESSING}

In this section we will review the data processing procedure employed to obtain $d \sigma / d \Omega$ in an absolute scale, and the resulting structure factor. The process is not straightforward due to the high inelasticity effects in the sample, which precludes the application of standard corrections to account for the departures froms the static approximation, under which the measured $S(Q)$ can be related to the liquid pair distribution function $g(r)$.

\section{A. Multiple-scattering and attenuation corrections}

The correction procedure for multiple-scattering and attenuation effects, as well as empty-cell subtraction (described in a previously published paper $^{8}$ ) is based on Monte Carlo simulations. It basically consists in the numerical solution of the equations developed by Sears ${ }^{9}$ along the line proposed by Copley. ${ }^{10}$ In the present work we will omit the details, giving only a general outline of the procedure. We will focus this description to the corrections performed on the hydrogen data, but the same procedure was applied to vanadium as explained in the following section.

Neutron histories randomly produced are followed individually. At each step the flight path is obtained randomly from a distribution governed by the total cross section as a function of the neutron energy calculated with the model developed by Granada. ${ }^{11}$ The distribution is biased in order that the neutron never leaves the sample. The energy after each scattering process is governed by Granada's synthetic scattering law, ${ }^{11}$ and the angular distributions are taken (in the first step) from the uncorrected experimental data. The output from each run serves as a correction for the angular distribution of the next run.

The calculated magnitudes are based on the macroscopic double-differential cross sections ${ }^{9}$

$$
\frac{d^{2} \Sigma}{d \Omega d E}=\frac{N}{4 \pi A} \frac{k}{k_{0}} s(Q, \varepsilon)
$$

where $N$ is the total number of scattering centers, $A$ the crosssectional area of the sample, $k_{0}$ and $k$ the (modulus of) the incident and emergent neutron wavevector, respectively, and $s(Q, \varepsilon)$ the effective scattering function. We symbolize with $\hbar Q$ the total impulse and with $\varepsilon$ the total energy exchanged in the sample after $n$ scattering processes. The effective scattering function admits a decomposition in a part due to neutrons that are singly scattered in the sample $s_{1}(Q, \varepsilon)$, singly scattered in the container $s_{c}(Q, \varepsilon)$ plus neutrons scattered in any combination of events in the sample and the container $s_{M}(Q, \varepsilon)$,

$$
s(Q, \varepsilon)=s_{1}(Q, \varepsilon)+s_{C}(Q, \varepsilon)+s_{M}(Q, \varepsilon) .
$$

The function $s_{1}(Q, \varepsilon)$ is simply related with the scattering law $S(Q, \varepsilon)$ through the relation

$$
s_{1}(Q, \varepsilon)=S(Q, \varepsilon) H(Q, \varepsilon),
$$

where $H(Q, \varepsilon)$ is the fraction of singly scattered neutrons that are not detected either due to multiple scattering and nuclear absorption processes or due to the detector nonideal efficiency.

The Monte Carlo algorithm records the angular distributions of the above defined magnitudes, i.e., their integrals at constant angle over all energy transfers. Thus

$$
\Sigma_{1, C, M}(\theta)=\left.\int_{\theta=\mathrm{const}} d E \frac{d^{2} \Sigma}{d \Omega d E}\right|_{1, C, M}
$$

where the subscripts indicate any of the above referred distributions. Also, we record the ideal angular distribution, i.e., those of singly scattered neutrons with no attenuation, which we call $\Sigma_{1}^{i d}(\theta)$, which allow us to define an angular attenuation factor

$$
H(\theta)=\frac{\Sigma_{1}(\theta)}{\sum_{1}^{i d}(\theta)},
$$

and a multiple-scattering factor

$$
f_{M S}(\theta)=\frac{\Sigma_{1}(\theta)}{\Sigma_{1}(\theta)+\Sigma_{C}(\theta)+\Sigma_{M}(\theta)}
$$

which serve as correction factors between successive iterations. Thus the corrected angular distribution to be applied in iteration $i+1$ is related to that employed in iteration $i$ through

$$
\sigma^{(i+1)}(\theta)=\sigma^{(i)}(\theta) \frac{f_{M S}^{(i)}(\theta)}{H^{(i)}(\theta)} .
$$


The iterative process finishes when no significant differences in the final angular distribution are observed.

Convergence was achieved after six iterations. In Fig. 1 we show the different components compared to experimental data and within the inset the attenuation factor as defined in Eq. (5) for an ideal detector efficiency which closely describes the behavior of the detectors at D1B at subthermal energies.

\section{B. Vanadium normalization}

Vanadium measurements were also corrected by multiplescattering and attenuation effects. The model employed in the numerical simulation to describe the energy transfers, angular distributions and total cross section was developed by Cuello and Granada. ${ }^{12}$ The absorption total cross-section data was described with the well-known " $1 / \mathrm{v}$ " law. ${ }^{13}$

We will describe the procedure employed to obtain the differential cross section in absolute units (barns/steradian) from the measured angular distributions in experimental units (counts/monitor). After the experimental angular distribution $I_{\text {exp }}(\theta)$ is corrected by attenuation and multiplescattering effects, a direct relation with the scattering power of the sample $S$ can be expressed as

$$
I_{\exp }^{\mathrm{tot}}=2 \pi \int_{0}^{\pi} I_{\exp }(\theta) \sin \theta d \theta=\alpha S,
$$

where $\alpha$ is a constant that links the experimental magnitude with the scattering power. We must notice that in order to calculate the integral, the integrand must be extrapolated to $180^{\circ}$. This can be safely done in the case of a quasi-isotropic scatterer such as vanadium. The scattering power can be readily calculated from

$$
S=1-T,
$$

where $T$ is the sample transmission coefficient for scattering processes, which for a cylinder sample (and normal incidence over the cylinder axis) is

$$
T=\frac{1}{2 R} \int_{-R}^{R} \exp \left[-2 n \sqrt{R^{2}-x^{2}} \sigma_{\text {scatt }}\left(E_{0}\right)\right] d x,
$$

where $R$ is the sample radius, $\sigma_{\text {scatt }}\left(E_{0}\right)$ the scattering total cross section at the incident neutron energy, and $n$ the number density of the sample.

At our incident energy $\sigma_{\text {scatt }}=8.89 \mathrm{~b},{ }^{14}$ so we obtain a scattering power of 0.391 from Eqs. (9) and (10). The application of Eq. (8) to our (corrected) experimental data gives a value of 0.3056 , so the value of $\alpha$ is 0.781 for our experimental setup. The normalized angular distribution $\widetilde{I}_{V}(\theta)$ is obtained imposing that its integral must equal $\sigma_{\text {scatt }}\left(E_{0}\right)$;

$$
2 \pi \int_{0}^{\pi} \widetilde{I}_{V}(\theta) \sin \theta d \theta=\sigma_{\text {scatt }}\left(E_{0}\right)
$$

so the required absolute normalization is

$$
\frac{d \sigma}{d \Omega}=\widetilde{I}_{V}(\theta)=\left[\frac{\sigma_{\text {scatt }}}{\alpha S_{V}}\right] I_{\text {exp }, V}(\theta) .
$$

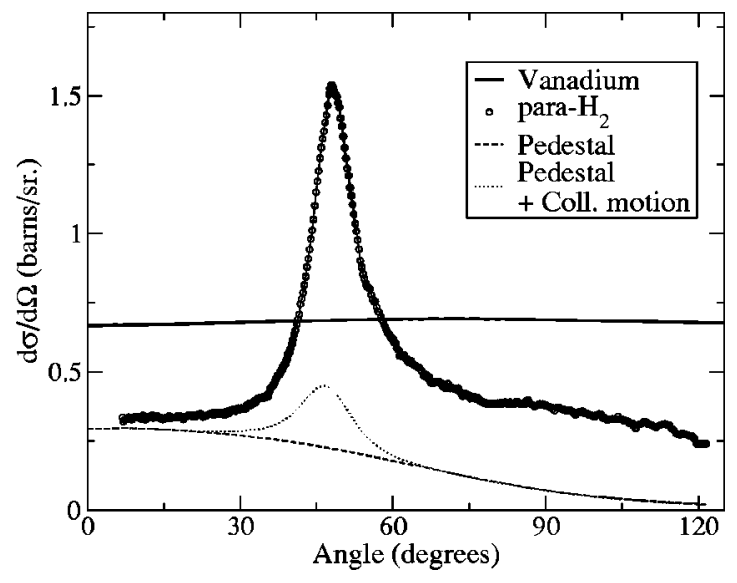

FIG. 2. Differential cross section of para- $\mathrm{H}_{2}$ vanadium in absolute units. The dashed line termed as "pedestal" is the contribution of the second and third terms of Eq. (13), whereas the dotted line labeled "pedestal + collective motion" includes the one-phonon effects described in Sec. IV A

The differential cross section thus obtained is shown in Fig. 2.

\section{Estimation of ortho-hydrogen contents}

The double-differential cross section per molecule of an ortho/para-hydrogen mixture, with a concentration $p_{O}$ of ortho-hydrogen for neutrons with energies below the rotational transition was calculated by Sears. ${ }^{15}$ The expression for $d \sigma / d \Omega$ (up to the first order in angular momentum transfers) can be derived from it as

$$
\begin{aligned}
\frac{d \sigma}{d \Omega}= & \frac{1}{4}\left\{\left(3 b^{(+)}+b^{(-)}\right)^{2}\left[j_{0}(Q r / 2)\right]^{2} S(Q)\right. \\
& +2 p_{O}\left(b^{(+)}-b^{(-)}\right)^{2}\left[j_{0}(Q r / 2)\right]^{2} f_{1}(Q) \\
& \left.+p_{O}\left(b^{(+)}-b^{(-)}\right)^{2}\left[j_{1}(Q r / 2)\right]^{2} f_{2}(Q)\right\},
\end{aligned}
$$

where $\quad b^{(+)}=1.0817 \times 10^{-14} \mathrm{~m} \quad$ and $\quad b^{(-)}=-4.742$ $\times 10^{-14} \mathrm{~m}$ are the proton-scattering lengths in the triplet and singlet states respectively, ${ }^{16} r=0.742 \AA$ is the mean distance between hydrogen nuclei in the molecule, ${ }^{15}$ and $j_{\ell}$ are the spherical Bessel functions of $\ell$-th order. $S(Q)$ is the molecular structure factor that in the limit $Q \rightarrow 0$ has the thermodynamic limit $0.054 .{ }^{17}$ The functions $f_{1}(Q)$ and $f_{2}(Q)$ are the form factors obtained by integration of the incoherent scattering law in the allowed kinematic range. Equation (13) allows us to assess the ortho-hydrogen contents of our sample by calculating its limit for $Q \rightarrow 0$, and comparing it with the experimental value. Thus $p_{O}$ can be obtained from the expression

$$
p_{O}=\frac{4 \frac{d \sigma}{d \Omega}(Q=0)-\left(3 b^{(+)}+b^{(-)}\right)^{2} S(0)}{2\left(b^{(+)}-b^{(-)}\right)^{2}} .
$$

On the basis of this equation, we employed an iterative scheme. In the first step it is assumed $p_{O}=0$, and multiple- 


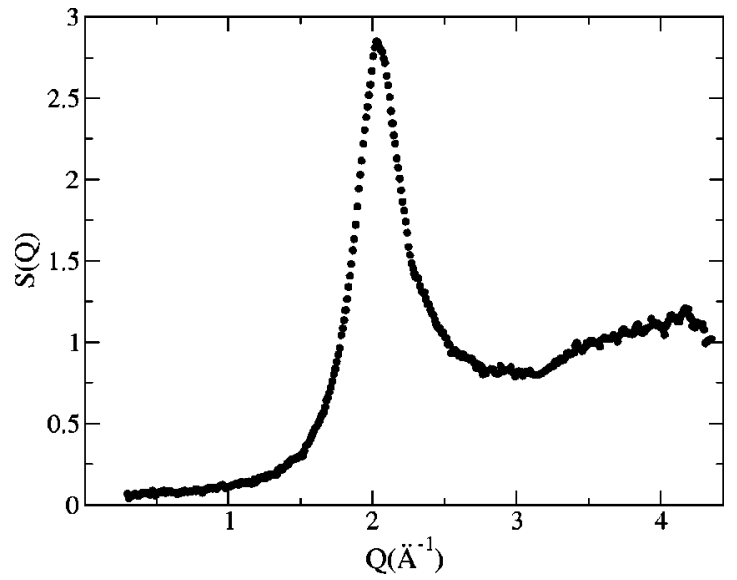

FIG. 3. Liquid para- $\mathrm{H}_{2}$ center-of-mass structure factor.

scattering and attenuation corrections are applied, as described in Sec. III A. We then obtained a value for $d \sigma / d \Omega(Q=0)$ that defined a value of $P_{O}$ from Eq. (14), and with this new value the corrections were recalculated. The process converged after two iterations, and thus we determined a content $p_{O}=0.017$ of ortho-hydrogen in our sample.

\section{Para-hydrogen differential cross section}

After applying the above described algorithm for multiple-scattering and attenuation corrections to the experimental data of liquid hydrogen, and refining the contents of ortho-hydrogen as described in the preceding section, we obtained the differential cross section by employing the vanadium calibration described in Sec. III B. Thus the differential cross section is determined by

$$
\frac{d \sigma}{d \Omega}=\widetilde{I}_{H}(\theta)=\left[\frac{\sigma_{\text {scatt }}^{H}}{\alpha S_{H}}\right] I_{\exp , H}(\theta),
$$

where the scattering cross section for our mixture at the incident neutron energy $\sigma_{\text {scatt }}^{H}=4.17 \mathrm{~b}$ is determined from Granada's model. ${ }^{11}$ Then, the scattering power of our sample is calculated as in Eqs. (9) and (10), thus obtaining $S_{H}=$ 0.097. The resulting $d \sigma / d \Omega$ is shown in Fig. 2.

\section{E. Center-of-mass structure factor}

Equation (13) can be applied to obtain $S(Q)$, the centerof-mass structure factor. The functions $f_{1}$ and $f_{2}$, are obtained from the integral of the incoherent scattering law over the allowed kinematic range of out experiment. ${ }^{15}$ Based on recoil scattering results ${ }^{18}$ we will describe the incoherent scattering law as that of a gas at $63 \mathrm{~K}$. In such a way, the second and third terms of Eq. (13) contribute with an inelastic pedestal as shown in Fig. 2. The resulting structure factor is shown in Fig. 3, which is the central result of the present paper. It can be compared to that derived from an inelastic neutron-scattering measurement previously reported. ${ }^{5}$

\section{DISCUSSION}

\section{A. Estimation of inelasticity corrections}

The experimental conditions under which the experiment has been carried out preclude the use of approximations usually employed in standard liquid diffraction work. In other words, the incident energy $E_{0}$ is, by force, far too low to enable the use of the static approximation that states that all final states are accessible, and therefore a measurement carried out under such conditions can provide a snapshot of the liquid structure.

In addition, account for inelasticity effects usually made by recourse to the Placzek expansion ${ }^{19}$ cannot be sensibly pursued since such an expansion about $\hbar \omega=0$ is only valid for small values of the ratio of neutron-to-particle masses $m_{n} / M$ and $k_{B} T / E_{0}$. In fact, the employed conditions are such that one can expect to find a relatively large inelasticity component to the interference term of the single-differential cross section since our sampling time will become comparable to the lifetime of the excitations. ${ }^{5}$

To our knowledge, there is no established route to estimate the magnitude of the correction referred to above. To proceed, we will make recourse to our previous knowledge of $S(Q, \omega)$ and calculate an inelasticity correction due to collective vibrations. We will estimate this following a heuristic way, starting from a one-phonon term. Multiphonon components are negligible at the present temperature and can hardly contribute to the total cross section, account made of the incident energy. In a previous paper the one-phonon contribution was successfully described by a damped harmonicoscillator function ${ }^{7}$

$$
I_{\text {inel }}(Q, \omega)=[n(\omega)+1] \frac{2 \omega Z_{Q} \omega_{Q} \Gamma_{Q}}{\left(\omega^{2}-\Omega_{Q}^{2}\right)^{2}+4 \omega^{2} \Gamma_{Q}^{2}},
$$

where $n(\omega)$ is the Bose occupation number, $\Gamma_{Q}$ the damping constant, $\Omega_{Q}$ the phonon frequency, $Z_{Q}$ a strength factor (proportional to the structure factor), and $\omega_{Q}$ obtained from $\Omega_{Q}^{2}=\omega_{Q}^{2}+\Gamma_{Q}^{2}$.

Throughout this section we will estimate the relative intensity that has to be assigned to the inelastic contribution derived from Eq. (16), when we add it to the one derived in Eq. (13). To this end we will assess the relative intensity of inelastic scattering starting from the total cross-section calculations.

To describe the different components of the total cross section we will base our calculations on the model proposed by Granada ${ }^{20}$, which describes the "self" cross section. The liquid is described with a free-translational molecular part and a vibrational intra-molecular one. Thus, if we follow the phonon expansion of the vibrational scattering law, we obtain for the zero-phonon term the expression

$$
S_{\text {self }}^{0}(Q, \omega)=\exp \left[-\gamma Q^{2}\right] S_{\text {gas }}(Q, \omega),
$$

where $S_{\text {gas }}(Q, \omega)$ is an ideal gas function of free-translating molecules of mass $M_{m o l}$ at temperature $T$ and $\gamma$ is a vibrational factor ${ }^{6}$ that has a value of $0.12 \AA^{2}$. The phonon components are obtained starting from the one-phonon term 

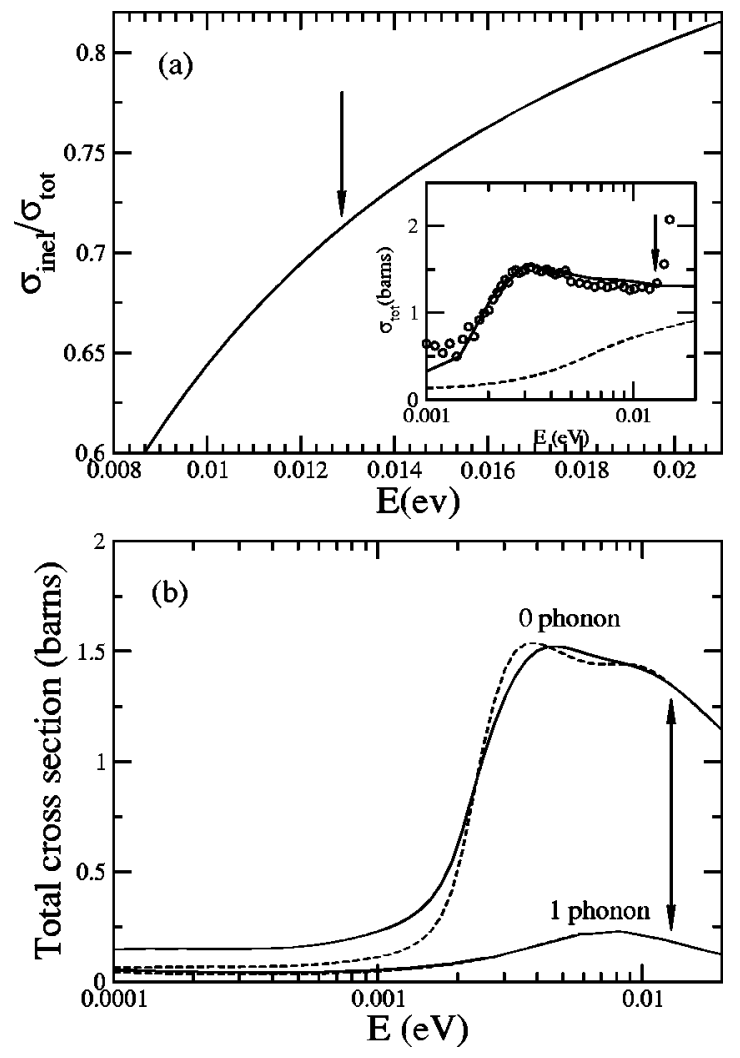

FIG. 4. (a) Ratio of inelastic to total scattering cross section for para-hydrogen. Inset: Seiffert's data (Ref. 3) compared with the present model (full line), and its inelastic component (dashed line). (b) Comparison of the result of using both the Vineyard and effective-mass model to calculate the one-phonon and the 0-phonon components to the total crosssection. The arrows indicate the incident neutron energy of the present diffraction experiment.

$$
\begin{aligned}
S_{\text {self }}^{1 p h}(Q, \omega)= & \frac{2 M}{\hbar} \gamma Q^{2} \exp \left[-\gamma Q^{2}\right] \\
& \times\left[\frac{Z(\omega)}{\omega}[n(\omega)+1] \otimes S_{\text {gas }}(Q, \omega)\right],
\end{aligned}
$$

where the density of vibrational states $Z(\omega)$ was taken from the above-mentioned model of para-hydrogen, ${ }^{11} M$ is the atomic hydrogen mass, and $n(\omega)$ is the Bose occupation number. The phonon terms are then obtained from iterated convolutions of this expression. Finally, the coherent behavior of para-hydrogen is described with the Vineyard approximation $^{21}$

$$
S(Q, \omega)=S(Q) S_{\text {self }}(Q, \omega)
$$

With the proposed model an accurate description of the experimental total cross section ${ }^{3}$ was achieved, ${ }^{11}$ as can be observed in the inset of Fig. 4(a), where the ratio of inelastic to the total scattering cross section is shown. The arrows in Fig. 4 show the incident neutron energy of our diffraction experiment. For $E_{0}=12.88 \mathrm{meV}$ a ratio of 0.71 of inelastic to total cross section is observed. It is worth mentioning that we employed different models of densities of states $Z(\omega)$ such as those mentioned in the above referred Granada's paper, ${ }^{11}$ and this ratio varied from 0.71 to 0.73 .

At this stage it is worth commenting on the validity of the Vineyard approximation as used in the present context. As a matter of fact, the approximation just referred to is known to be rather crude and does not predict the correct second moment of the scattering function. As a consequence it does not reproduce the de Gennes narrowing effect of the $Q$-dependent frequency spectrum for wave vectors corresponding to the first diffraction maximum.

The reliability of such an approximation for the purposes herein pursued was checked by means of numerical calculations using an alternative model that accounts for such a narrowing in terms of an effective mass that has an explicit $Q$ dependence given by $S(Q)$. Figure 4(b) shows that one basically gets the same results whether the Vineyard approximation or a more sophisticated model is used. This comes as a consequence of the kinematic conditions used in the present experiment as well as from the integral nature of the property we aim to calculate (i.e., the ratio of total to inelastic scattering). In fact, for our incident energy the integration range (in the energy-transfer variable $\hbar \omega$ ) comprising the relevant scattering law from where the total cross section is calculated extends up to $12 \mathrm{meV}$ for $Q=2 \AA^{-1}$. If a function that includes the de Gennes narrowing is used instead of the Vineyard approximation we obtain a distribution in $\hbar \omega$ that while preserving the area limits its range to lower energies. However since the integral over such frequency distribution is the figure of merit, we obtain basically the same results in both cases.

Having established the ratio of inelastic/total scattering we return to our diffraction experiment and calculate the intensity of the total and inelastic cross sections. Our line of reasoning is based upon Vineyard's picture. In the first place we must notice that the total scattering cross section can be obtained by inserting the experimental $d \sigma / d \Omega$ in Eq. (11). To calculate the inelastic intensity, we have to proceed carefully, since the following two different contributions are identified.

(a) The contribution arising from single-molecule form factors given by Eq. (13) plus the one derived from the collective motion [Eq. (16)].

(b) An additional correction that needs to be made to account for the falloff at large $Q$ and arises mostly from large recoil effects.

Since both contributions described in (a) have an unknown relative weight, we have introduced a free parameter to describe it. The inelastic component (b) is understood in the Vineyard picture [Eqs. (17) and (19)] as follows: if all the scattering were elastic then the static approximation would hold and therefore $S^{e l}(Q, \omega)=S(Q) \delta(\omega)$. However, recoil and zero-point vibration effects are in operation and these are accounted for by a factor $\exp \left(-\gamma Q^{2}\right) S_{g a s}$ in the zero-phonon term. Therefore the inelastic component (b) is the experimental $d \sigma / d \Omega$ minus the contributions included in (a), weighed by the factor $\left[1-\exp \left(-\gamma Q^{2}\right) S_{\text {gas }}\right]$. After having established the inelastic components we calculate their ratio to the total scattering as a function of the above-mentioned free parameter. At the end of this process we obtain the inelastic 
contribution depicted in Fig. 2 that includes the collective motions. The resulting relative weight of the inelastic components is 0.85 for the single-molecule form factors given by Eq. (13) plus 0.15 for the one derived from the collective motion [Eq. (16)].

\section{B. Comparison to path-integral-molecular-dynamics results}

The renewed interest in the determination of the static structure factor of liquid para- $\mathrm{H}_{2}$ has been motivated by the advent of computer simulations based upon path-integral representations that allow a realistic account of the quantum degrees of freedom. Here we set ourselves to compare the present experimental results with those arising from a recent simulation carried out using the PICMD approach. ${ }^{22-24}$ It uses a path-integral representation for a quasiclassical variable named the centroid, which is the average position of the center of the spatial dispersion of the quantum particles. The latter are treated as semiclassical Boltzmann particles and their Newtonian equations of motion are integrated following conventional MD procedures. ${ }^{25}$ Within the PICMD formalism, the state of the set of $N$ quantum particles is specified by the partition function given in terms of a path-integral representation which is assimilated to the configurational integral for $N$ classical ring polymers, each one of those representing the path of an individual particle or "bead" along the imaginary-time axis. The centroid is thus defined as the average position of the Feynman path, or equivalently as the position of the center of mass of the isomorphous polymer. The dynamics which follows Newton laws is driven by forces generated by a potential $V_{c}(r)$ which is averaged over all the quantum degrees of freedom. In more explicit terms, the interparticle potential can basically be regarded as the bare interatomic interaction which is renormalized by the quantum fluctuation. ${ }^{23}$ As recently shown, the centroid correlation functions can be related to semiclassical approximations. ${ }^{26}$ While analytic continuation of exact imaginary-time path integral Monte Carlo techniques ${ }^{27}$ and quantum versions of the mode-coupling theory ${ }^{28}$ are at present under development, PICMD provides a computationally convenient framework to calculate real-time properties of the condensed phases of many-body systems.

The Silvera-Goldman form is chosen ${ }^{5,6}$ to represent the bare interaction potential and the simulation was run for an ensemble of 500 molecules with periodic boundary conditions at $T=15 \mathrm{~K}$ and a pressure of $1 \times 10^{5} \mathrm{~Pa}$ that yield a molar volume of $24.513 \mathrm{~cm}^{3} \mathrm{~mol}^{-1}$. The statistical ensemble corresponds to isobaric-isothermal conditions and the simulation method followed the Nose-Hoover-chain-Andersentype NPT scheme. The simulation was run for 100000 steps that amount to $25 \mathrm{ps}$.

Our previous estimate for $S(Q)$ was derived by integration of the measured $S(Q, \omega)$ dynamic structure factor ${ }^{5}$ over a limited range of energy transfers, that is,

$$
S(Q)=\int_{E_{\min }}^{E_{\max }} d \omega S(Q, \omega),
$$

with $E_{\max }=10 \mathrm{meV}$, in order to avoid contamination arising from the para $\rightarrow$ ortho rotational peaks. It yielded a value of

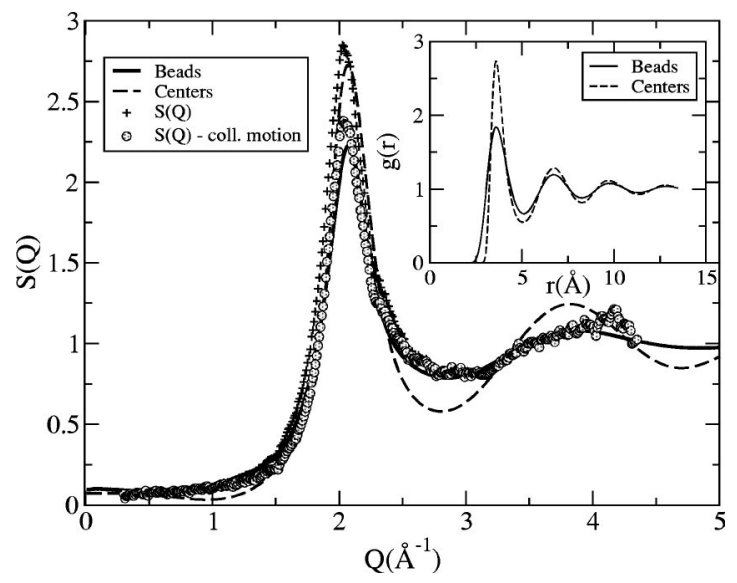

FIG. 5. Results from the present experiment compared with the PICMD simulations (see text for details). The inset shows the $g(r)$ radial distribution for both cases.

$S(Q)$ at the peak maximum of 2.83 well in excess of that reported for liquid hydrogen by Zoppi et al. ${ }^{29}$ and even above that of about 2.25 reported for liquid deuterium. ${ }^{17}$ Our present data displayed in Fig. 3 show a peak with a height that is again in excess of those arising from conventional diffraction measurements and showing good agreement with our previous estimate. ${ }^{5}$ In both cases, the dominant excitation being explored is the longitudinal phonon, and under such conditions a very significant part of the scattering is inelastic, as can be gauged by comparison of the individual spectra previously reported. ${ }^{7}$

Figure 5 compares the present experimental result with the structure factors arising from the PICMD simulations. These correspond to the structure factors for the centroids $S_{\text {centroids }}(Q)$, and beads $S_{\text {beads }}(Q)$, respectively, that as mentioned above represent the average position of Feynman path and the individual positions of the nodes along such path. As seen there is a noticeable difference between the height of the main peak of both structure factors as well as with the decay of the oscillation. The height of both calculated structure factors differ by a significant amount. That corresponding to the $S_{\text {beads }}(Q)$ function reaches 2.23 while that from $S_{\text {centroids }}(Q)$ yields a significantly higher value of 2.73. Both cases depict a significantly structured liquid as oscillations in the calculated radial distributions show in the inset of Fig. 5 that persist up to $14 \AA$. The height and shape of the main peak of the $S_{\text {centroids }}(Q)$ structure factor comes rather close to experiment up to $Q \approx 2.5 \AA^{-1}$, while the second oscillation in the simulated quantity becomes far more marked than experiment. Subtraction of the inelasticity contribution due to collective excitations referred to above yields a structure factor that is in rather good agreement with $S_{\text {beads }}(Q)$ since now both oscillations are well matched.

The comparison just referred to above clarifies the discrepancies between measurements carried out under very different conditions. As shown in a previous paper ${ }^{6}$ the estimates for the quantity directly accessible from simulations such as the $g(r)$ radial distribution are in very good agreement irrespective of the method of calculation. In other 
words, $S_{\text {beads }}(Q)$ compares well to the quantity obtained from path-integral monte carlo simulations. Experimental determination of such radial distributions requires the static approximation to hold or, in other words, a sampling time short enough so that a true static picture of the liquid structure can be made. Such conditions cannot be matched in neutron work for reasons explained at the beginning, and use of X-ray beams will only provide an estimate for $g(r)$ about one order of magnitude less accurate than neutrons. ${ }^{19}$

On the other hand, one expects $S_{\text {centroids }}(Q)$ to match the result measured using low incident energies since its dynamic counterpart $S_{\text {centroids }}(Q, \omega)$ was found to be in good agreement with experimental spectra, ${ }^{5,6}$ and therefore it

*Electronic address: javier@cab.cnea.gov.ar

${ }^{\dagger}$ Also at Consejo Superior de Investigaciones Científicas, Instituto de Estructura de la Materia, Serrano 123 E-28006 Madrid, Spain.

${ }^{1}$ M. Ross Rep. Prog. Phys. 48, 1 (1985).

${ }^{2}$ I.F. Silvera, Rev. Mod. Phys. 52, 393 (1980); J.-L. Li, G.-M. Rignanese, E.K. Chang, X. Blase, and S.G. Louie, Phys. Rev. B 66, 035102 (2002); E. Gregoryanz, A.F. Goncharov, K. Matsuishi, H.-K. Mao, and R.J. Hemley, Phys. Rev. Lett. 90, 175701 (2003); N.A. Tahir et al., Phys. Rev. B 67, 184101 (2003); R. Redmer, G. Röpke, S. Kuhlbrodt, and H. Reinholz, ibid. 63, 233104 (2001); W.J. Nellis, S.T. Weir, and A.C. Mitchell, ibid. 59, 3434 (1999); M. Ross, ibid. 54, R9589 (1996); S.T. Weir, A.C. Mitchell, and W.J. Nellis, Phys. Rev. Lett. 76, 1860 (1996); I.I. Mazin and R.E. Cohen, Phys. Rev. B 52, R8597 (1995).

${ }^{3}$ W.D. Seiffert, Report No. EUR 4455d, 1970 (unpublished).

${ }^{4}$ J.A. Young and J.U. Koppel, Phys. Rev. 135, A603 (1964).

${ }^{5}$ F.J. Bermejo, K. Kinugawa, C. Cabrillo, S.M. Bennington, B. Fåk, M.T. Fernández-Díaz, P. Verkerk, J. Dawidowski, and R. Fernández-Perea, Phys. Rev. Lett. 84, 5359 (2000).

${ }^{6}$ F.J. Bermejo, B. Fåk, S.M. Bennington, K. Kinugawa, J. Dawidowski, M.T. Fernández-Díaz, C. Cabrillo, and R. FernándezPerea, Phys. Rev. B 66, 212202 (2002).

${ }^{7}$ F.J. Bermejo, B. Fåk, S.M. Bennington, R. Fernández-Perea, C. Cabrillo, J. Dawidowski, M.T. Fernández-Díaz, and P. Verkerk, Phys. Rev. B 60, 15154 (1999).

${ }^{8}$ J. Dawidowski, F.J. Bermejo, and J.R. Granada, Phys. Rev. B 58, 706 (1998).

${ }^{9}$ V.F. Sears, Adv. Phys. 24, 1 (1975).

${ }^{10}$ J.R.D. Copley, Comput. Phys. Commun. 7, 289 (1974); J.R.D. Copley, P. Verkerk, A.A. Van Well, and H. Fredrikze, ibid. 40, 337 (1986).

${ }^{11}$ J.R. Granada, V.H. Gillette, M.E. Pepe, and M.M. Sbaffoni, J. Neutron Res. 11, 25 (2003). should come as no surprise that the quantity derived by recourse to Eq. (20) in an inelastic-scattering measurement ${ }^{5}$ matches the present result shown in Fig. 3.

Finally, the present set of data are at variance with those reported by Zoppi et al..$^{29}$ which were derived from hot neutron diffraction. As referred to above and already explained in detail, ${ }^{6}$ the difference in both measurements cannot explained only be by the rather different conditions employed in both experiments. In fact, the liquid peak becomes barely visible in the single-differential cross section reported in Ref. 29 , and therefore the systematic accuracy claimed by Zoppi et $a .^{29}$ in their estimate of $S(Q)$ which yields a peak height somewhat below 2.0, seems grossly exaggerated.

${ }^{12}$ G.J. Cuello and J.R. Granada, Ann. Nucl. Energy 24, 763 (1997).

${ }^{13}$ S.F. Mughabghab, M. Divadeenam, and N.E. Holden, Neutron Cross Sections (Academic Press, New York, 1981), Vol. 1, pt. B.

${ }^{14}$ A. Smith, D. Smith, P. Guenther, J. Meadows, R. Lawson, T. Djemil, and R. Howeton, ENDF/B-VI, IAEA Nuclear Data Section Release 8 (2001).

${ }^{15}$ V.F. Sears, Can. J. Phys. 44, 1279 (1966).

${ }^{16}$ L. Koester, H. Rauch, and E. Seymann, At. Data Nucl. Data Tables 49, 65 (1991).

${ }^{17}$ M. Zoppi, M. Celli, and A.K. Soper, Phys. Rev. B 58, 11905 (1998).

${ }^{18}$ W. Langel, D.L. Price, R.O. Simmons, and P.E. Sokol, Phys. Rev. B 38, 11275 (1988).

${ }^{19}$ P.A. Egelstaff, in Methods Of Experimental Physics (Academic Press, New York, 1987), Chap. 14, p. 428.

${ }^{20}$ J.R. Granada, Phys. Rev. B 31, 4167 (1985).

${ }^{21}$ G.H. Vineyard, Phys. Rev. 110, 999 (1958).

${ }^{22}$ K. Kinugawa, Chem. Phys. Lett. 292, 454 (1998); K. Kinugawa, P.B. Moore, and M.L. Klein, J. Chem. Phys. 106, 1154 (1997).

${ }^{23}$ S. Miura, S. Okazaki, and K. Kinugawa, J. Chem. Phys. 110, 4523 (1999).

${ }^{24}$ J. Cao and G.A. Voth, J. Chem. Phys. 101, 6168 (1994); 101, 6157 (1994).

${ }^{25}$ G.J. Martyna, M.E. Tuckerman, D.J. Tobias, and M.L. Klein, Mol. Phys. 87, 1117 (1996).

${ }^{26}$ Q. Shi and E. Geva, J. Chem. Phys. 118, 8173 (2003).

${ }^{27}$ E. Rabani, D.R. Reichman, G. Krilov, and B.J. Berne, Proc. Natl. Acad. Sci. U.S.A. 99, 1129 (2002).

${ }^{28}$ E. Rabani and D.R. Reichmann, Europhys. Lett. 60, 656 (2002).

${ }^{29}$ M. Zoppi, M. Celli, and A.K. Soper, Phys. Rev. B 58, 11905 (1998); See also M. Celli, U. Bafile, G.J. Cuello, F. Formisano, E. Guarini, R. Magli, M. Neumann, and M. Zoppi, cond-mat/0209239 (unpublished). 